\title{
Modélisation numerique et expérimentale du comportement de structures sandwich
}

\author{
Moussa Karama $^{\text {a }}$ ET Bernard Lorrain \\ École Nationale d'Ingénieurs de Tarbes, Équipe CMAO, Groupe M² SF, 47 avenue d'Azereix, BP 1629, \\ 65016 Tarbes Cedex, France
}

Reçu le 10 juillet 2003, accepté le 8 juillet 2005

\begin{abstract}
Résumé - Le but de ce travail dans le thème « Modélisation de structures composites » est la modélisation en flexion de structures sandwiches. Les étapes principales de cette étude sont : la détermination des constantes élastiques du matériau par une homogénéisation numérique à l'aide du logiciel « Samcef »; le développement analytique et l'implémentation dans le logiciel de calcul formel « Mathematica » de modèles raffinés de la flexion 3 et 4 points; la validation expérimentale des différents modèles développés. Il est important de noter la mise en œuvre de nouveaux moyens de mesure tels que la stéréocorrélation d'images et l'interférométrie laser pour la mesure de déformations, des déplacements et des contraintes.
\end{abstract}

Mots clés : Matériaux sandwich / homogénéisation / modélisation analytique / flexion / validation expérimentale

\begin{abstract}
Experimental and numerical modelling of the behaviour of sandwich materials. The aim of this work concerning the research theme "Modelling of composite structures" is to model the bending behaviour of sandwich structures. The main steps in this study are: the determination of the elastic constants of the material by numerical homogenisation "Samcef"; the analytical development of 3 and 4-point refined bending models and their implementation in formal calculation software "Mathematica"; the numerical simulation of the overall behaviour of the material in order to draw up a reference solution "Samcef"; the experimental validation of the various models developed. It is important to note the use of new means of measurement such as stereovision (based on image correlation) and laser interferometry in strain, displacement and stress measurement.
\end{abstract}

Key words: Sandwich structures / homogenization / analytical modelling / bending / experimental validation

\section{Introduction}

Le monde industriel est amené à une utilisation de plus en plus importante des matériaux composites en raison de leur grande souplesse en construction mécanique, de leur niveau de résistance élevé pour une masse volumique faible et grâce à l'évolution récente des techniques de fabrication.

Les matériaux sandwiches à structure nid d'abeilles sont un bon compromis entre résistance et légèreté et possèdent d'excellentes caractéristiques d'isolation thermique. Étant donné le coût de l'utilisation de ces matériaux pour des structures, il est intéressant de connaître leurs propriétés mécaniques pour prédire et

${ }^{a}$ Auteur correspondant : moussa@enit.fr calculer leur comportement dans des environnements spécifiques et variés.

Sous sa forme la plus générale, le problème du dimensionnement d'une structure sandwich consiste à chercher le matériau sandwich nécessaire pour que la structure réponde à un cahier des charges donné. Le problème peut être résolu par diverses solutions qui diffèrent par la nature du matériau sandwich retenu : nature de l'âme, nature des peaux, épaisseur du matériau, etc. Dans la pratique, différentes considérations conduiront à la solution finale, par exemple : masse minimale, processus de fabrication imposé, matériaux donnés, conditions d'utilisation de la structure, etc.

Le problème du prédimensionnement peut être abordé soit par des méthodes analytiques soit par des méthodes numériques. Les méthodes analytiques conduisent à des résultats généraux, permettant de dégager l'influence des 
divers paramètres. Ces méthodes sont toutefois limitées à l'analyse de structures simples : poutres, plaques, coques cylindriques, etc. Dans le cas de structures complexes, l'analyse par éléments finis s'impose comme l'outil usuel du concepteur. L'analyse nécessite alors trois étapes :

- La détermination des caractéristiques mécaniques constituant le matériau sandwich.

- La validation du modèle utilisé pour décrire le comportement mécanique du matériau sandwich, ainsi que la validation de l'élément fini associé.

- Le dimensionnement lui-même.

L'objet de ce travail est de développer cette démarche dans le cas de structures sandwiches, pour évoquer le particularisme de ces matériaux et dresser l'état de l'art en la matière.

\section{Approche prévisionnelle des caractéristiques du nid d'abeilles « nida »}

Les matériaux sandwiches sont composés de plusieurs parties interagissant entre elles. On trouve donc les peaux ou semelles ayant des caractéristiques mécaniques propres et le cœur ou âme pouvant être entre autres du nid d'abeilles (nida) ayant des propriétés mécaniques spécifiques. L'assemblage de ces deux parties se réalise par collage, soudage ou brasage mettant en jeu un autre corps de comportement différent.

L'intérêt de l'étude est de pouvoir homogénéiser le nida indépendamment des peaux, en remplaçant le nida de géométrie compliquée par un matériau homogène de propriétés mécaniques similaires dans les modèles analytiques et numériques. Le nid d'abeilles est assimilable à un matériau orthotrope. Son homogénéisation passe tout d'abord par la détermination des constantes élastiques de l'ingénieur qui sont les modules d'élasticité, les coefficients de Poisson et les modules de cisaillement.

La connaissance du comportement mécanique des âmes, en particulier en cisaillement, est évidemment indispensable lors de la phase de conception des structures sandwiches. L'estimation de ce comportement consiste à s'appuyer sur la structure répétitive des nids d'abeilles pour réaliser des calculs d'homogénéisation. On peut alors prédire de nombreux coefficients élastiques $[1,2]$. Toutefois, dans le cas général, les modules de cisaillement peuvent seulement être bornés avec ce type d'approche [3].

\subsection{Géométrie et notations}

Il est à noter que suivant les littératures, les dénominations et indices peuvent varier. La géométrie ainsi que les notations utilisées par la suite sont détaillées ci-après.

- les modules d'élasticité : $E_{i}$, où $i$ est la direction de traction ;
- les coefficients de Poisson : $\nu_{i j}$, où $i$ est la direction de traction et $j$ est la direction de la contraction;

- les modules de cisaillement : $G_{i j}$ module de cisaillement dans le plan $(i, j)$.

Un nid d'abeilles peut être caractérisé par un angle de cellule et quatre paramètres définis à partir des caractéristiques suivantes :

$\theta$ angle de cellule,

$\phi$ diamètre de cellule,

$b$ longueur d'une paroi centrale,

$a$ longueur d'une paroi inclinée,

$t^{\prime}$ épaisseur d'une paroi centrale,

$t$ épaisseur d'une paroi inclinée.

$h$ hauteur du nid d'abeilles.

\subsection{Principales caractéristiques élastiques du nida}

Le nid d'abeilles peut être assimilé à un matériau orthotrope. Pour le caractériser, il faut donc déterminer les neuf constantes de l'ingénieur :

- les modules d'élasticité $E_{1}, E_{2}, E_{3}$,

- les coefficients de Poisson $\nu_{12}, \nu_{23}, \nu_{13}$

- les modules de cisaillement $G_{12}, G_{23}, G_{13}$.

Afin de compléter cette étude, nous avons également déterminé (sans utiliser les relations de symétrie), les constantes suivantes : $\nu_{21}, \nu_{32}, \nu_{31}, G_{21}, G_{32}, G_{31}$.

La détermination de ces caractéristiques mécaniques peut être effectuée grâce à deux méthodes différentes :

- la modélisation analytique,

- la modélisation numérique.

En comparant les résultats obtenus à partir de ces deux méthodes, on peut ainsi effectuer une approximation des constantes de l'ingénieur du nida.

\subsubsection{Approches numériques}

L'objectif est la détermination des constantes élastiques par une méthode d'homogénéisation numérique appliquée à l'échelle d'un volume élémentaire représentatif du nid d'abeilles afin de comparer les résultats avec ceux obtenus analytiquement.

Neuf simulations sont nécessaires pour déterminer les neuf constantes :

- trois simulations de traction simple, chacune permettant de calculer un module d'élasticité et deux coefficients de Poisson;

- six simulations de cisaillement, une pour chaque module de cisaillement.

Les propriétés mécaniques du nida étant intimement liées à ses caractéristiques géométriques, il est important de bien les définir. 


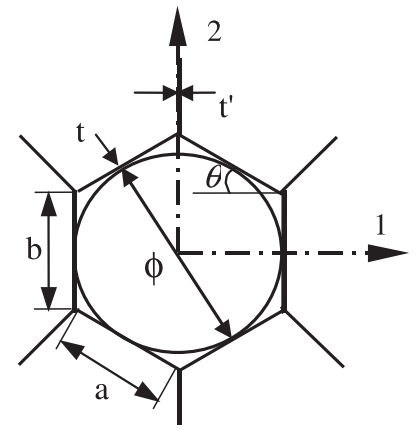

Fig. 1. Géométrie d'un nid d'abeilles.

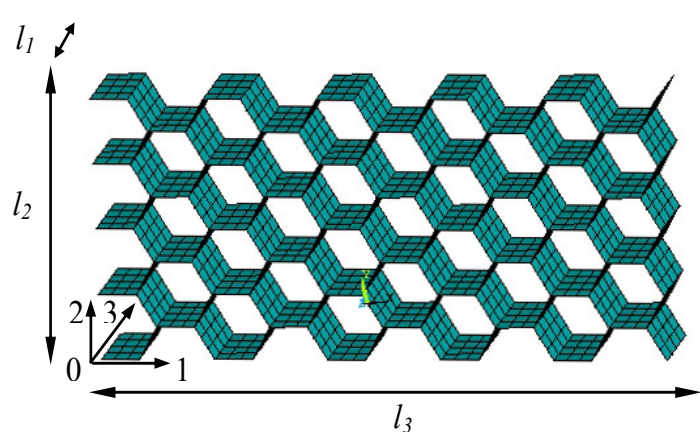

Fig. 2. Volume élémentaire représentatif « V.E.R. ».

\subsubsection{Le volume élémentaire représentatif « V.E.R. »}

Il est nécessaire pour des raisons de temps de calcul et de puissance de calcul disponible de réduire les modèles et donc d'utiliser les symétries du modèle.

Dans le cas du nida, le nombre de modèles pouvant être créés est abondant. Les modèles utilisés par la suite sont guidés par les études et les simulations effectuées sur le nid d'abeilles par de nombreuses équipes de recherche [3-5] afin de pouvoir au préalable comparer les résultats des différentes simulations, améliorer et adapter si possible les modèles au code de calcul Samcef [6,7].

La géométrie est à base de cellules hexagonales régulières. Si l'on se réfère aux notations de la figure 1, les caractéristiques sont les suivantes : $\phi=6,35 \mathrm{~mm}$, $a=b=3,66 \mathrm{~mm}, h=9,525 \mathrm{~mm}, \theta=30^{\circ}$. Les longueurs $l_{1}, l_{2}, l_{3}$ du volume élémentaire représentatif sont (Fig. 2) : $l_{1}=54,986 \mathrm{~mm}, l_{2}=25,4 \mathrm{~mm}, l_{3}=9,525 \mathrm{~mm}$. L'épaisseur $t$ est paramétrée. Pour ce modèle, toutes les simulations sont effectuées avec quatre valeurs de $t$ et la double épaisseur à la liaison entre deux cellules adjacentes est prise en compte. Le matériau considéré est de l'aluminium 5056 avec les caractéristiques suivantes : $E_{0}=68,940 \times 10^{3} \mathrm{MPa}, G=6,54 \times 10^{3} \mathrm{MPa}, \nu=0,312$.

\subsubsection{La méthode d'homogénéisation}

Les simulations ont été effectuées sur le code de calcul Samcef. Le modèle de nida prend en compte 40 cellules qui ont été maillées avec des éléments plaques à 4 nœuds à 6 degrés de liberté par nœud.

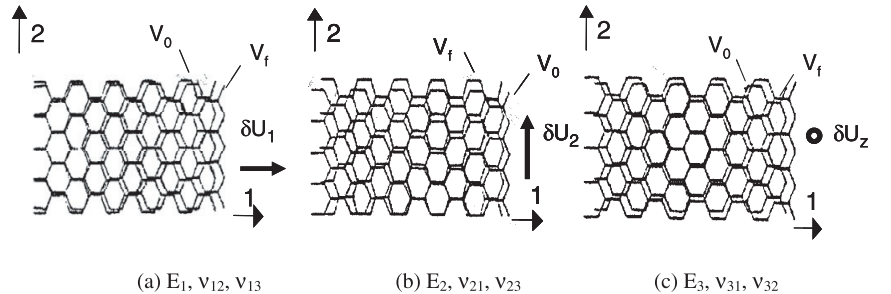

Fig. 3. V.E.R. en déplacement imposé suivant 1 (a); 2 (b); 3 (c).

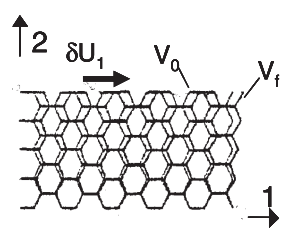

(d) $\mathrm{G}_{12}=\tau_{12} / \gamma_{12}$

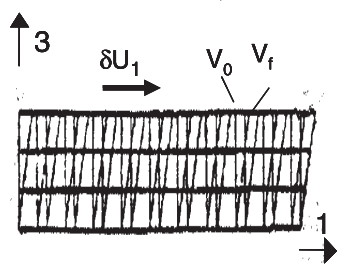

(f) $\mathbf{G}_{13}=\tau_{13} / \gamma_{13}$

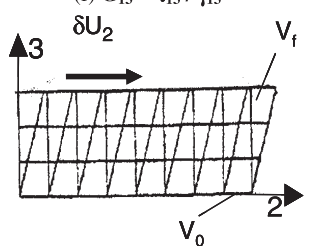

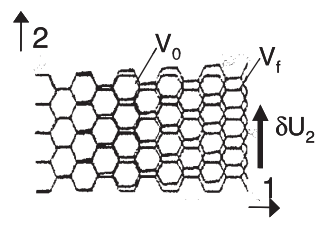

(e) $\mathrm{G}_{21}=\tau_{21} / \gamma_{21}$

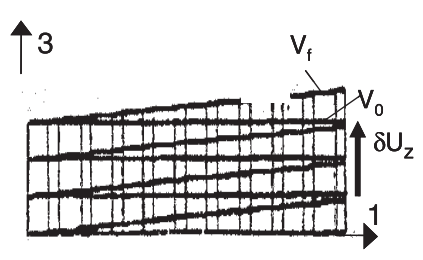

(g) $\mathbf{G}_{31}=\tau_{31} / \gamma_{31}$

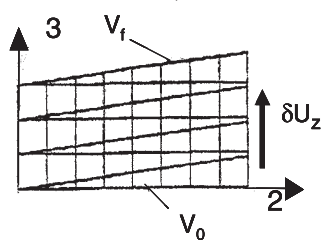

Fig. 4. V.E.R. sous sollicitation de cisaillement (d-i).

Pour ce modèle, le maillage choisi permet de ne pas trop alourdir le calcul tout en gardant une bonne précision au niveau des résultats. Un raffinement excessif ne donne pas de meilleurs résultats donc le maillage retenu compte 1500 éléments et 1725 nœuds. Chaque face de l'hexagone (clinquant) est discrétisée par 12 éléments, 4 suivant l'axe 3 et 3 suivant l'axe 1.

Pour calculer les différents modules, un déplacement est imposé sur une face du V.E.R. dans une direction donnée, la face opposée étant fixe. Les symétries sont prises en compte au niveau des conditions limites. Neuf simulations sont nécessaires pour déterminer les neuf constantes élastiques du nida :

- La simulation d'une sollicitation de traction suivant une direction $i(i=1,2,3)$ permet de déterminer les 3 modules de rigidité $E_{1}, E_{2}$ et $E_{3}$ et les 6 coefficients de Poisson (Figs. 3a-c). $V_{0}$ : volume avant déformation, $V_{\mathrm{f}}$ : volume après déformation.

- La simulation des sollicitations de cisaillement suivant les plans 12, 23, 13 (2 simulations par plan) permet de déterminer les 6 coefficients de cisaillement (Figs. 4d-i).

Cette méthode basée sur l'analyse des efforts aux liaisons, à partir d'un déplacement imposé, permet par 
Tableau 1. Résultats de la traction suivant 1.

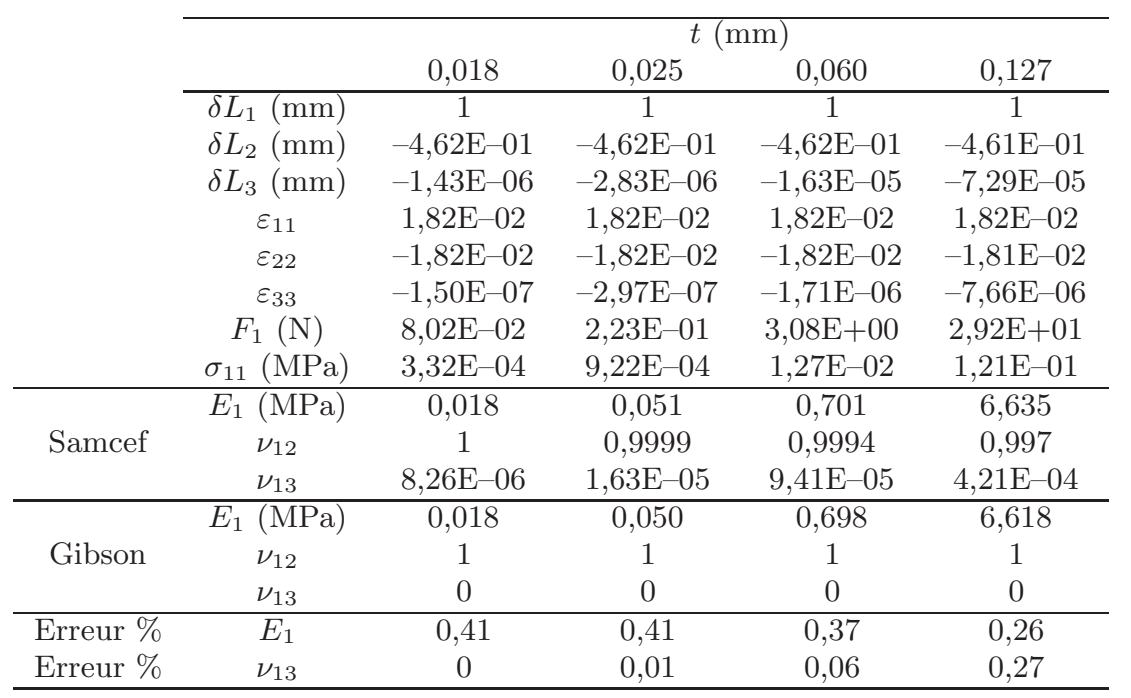

l'utilisation des lois de comportement de déduire les constantes élastiques.

\subsubsection{Quelques résultats}

\subsection{Homogénéisation des modules de Young et des coefficients de Poisson}

Le tableau 1 présente les résultats obtenus par simulations numériques pour une série de conditions aux limites (déplacement imposé suivant la direction 1) et pour 4 épaisseurs (t) du feuillard et les compare aux valeurs analytiques obtenues par les formules de Gibson suivantes :

$$
\begin{aligned}
E_{1} & =E_{o} \cdot\left(\frac{t}{a}\right)^{3} \cdot \frac{\cos \theta}{(b / a+\sin \theta) \cdot \sin ^{2} \theta} \\
\nu_{21} & =\frac{(b / a+\sin \theta) \cdot \sin \theta}{\cos ^{2} \theta} \\
\nu_{12} & =\frac{\cos ^{2} \theta}{(b / a+\sin \theta) \cdot \sin \theta}
\end{aligned}
$$

On peut remarquer que l'écart des résultats entre la simulation numérique et le modèle analytique est faible puisque l'erreur maximale pour $E_{1}$ est de 0,4\%. L'erreur sur les coefficients de Poisson est très faible.

\subsection{Homogénéisation des modules de cisaillement}

Dans cette méthode comme les précédentes, les caractéristiques en cisaillement sont déterminées par l'analyse des efforts aux liaisons en utilisant les lois de comportement en hypothèse de petites perturbations.

Le module de cisaillement est défini par la relation :

$$
G_{i j}=\frac{\sigma_{i j}}{\gamma_{i j}}, \quad i \neq j \text { et } i, j=1,2,3 .
$$

Tableau 2. Résultats en cisaillement pour un déplacement imposé suivant la direction $z$.

\begin{tabular}{cccccc}
\cline { 2 - 6 } & & \multicolumn{5}{c}{$t(\mathrm{~mm})$} \\
& $G_{23}$ & 0,018 & 0,025 & 0,060 & 0,127 \\
\cline { 2 - 6 } & $\delta L_{1}(\mathrm{~mm})$ & 0,1 & 0,1 & 0,1 & 0,1 \\
& $\gamma_{23}$ & 0,011 & 0,011 & 0,011 & 0,011 \\
& $F_{2}(\mathrm{~N})$ & 1078,9 & 1517 & 3641,2 & 7707,6 \\
& $\sigma_{23}(\mathrm{MPa})$ & 0,77 & 1,09 & 2,61 & 5,52 \\
\hline Samcef & $G_{23}(\mathrm{MPa})$ & 73,57 & 103,45 & 248,30 & 525,59 \\
Gibson & $\mathrm{G}_{23}(\mathrm{MPa})$ & 74,31 & 104,49 & 250,77 & 530,80 \\
Erreur $\%$ & $\mathrm{G}_{23}$ & 1 & 1 & 0,99 & 0,98 \\
\hline
\end{tabular}

avec $\sigma_{i j}=\frac{F_{i}}{S_{i k}}$ la contrainte de cisaillement, $S_{i k}=l_{i} l_{k}$ surface de la face fixée et $\gamma_{i j} \approx \tan \gamma_{i j}=\frac{\delta U_{i}}{l_{j}}$ la déformation de cisaillement. $F_{i}$ est déterminée par la simulation numérique.

Les formes analytiques des différents modules sont données par les équations (5) à (7) [1,3].

$$
G_{12}=E_{o} \cdot\left(\frac{t}{a}\right)^{3} \cdot \frac{b / a+\sin \theta}{(b / a)^{2} \cdot(1+b / 4 a) \cdot \cos \theta}
$$

$$
\begin{aligned}
& G \cdot\left(\frac{t}{a}\right) \cdot \frac{b / a+\sin \theta}{(1+b / a) \cdot \cos \theta} \leq G_{13} \\
& \leq G \cdot \frac{b / a+\sin ^{2} \theta}{\cos \theta \cdot(b / a+\sin \theta)} \cdot\left(\frac{t}{a}\right) \\
& G_{23}=G \cdot \frac{\cos \theta}{b / a+\sin \theta} \cdot\left(\frac{t}{a}\right)
\end{aligned}
$$

Le tableau 2 présente pour 4 épaisseurs de feuillard les résultats obtenus pour le module de cisaillement $G_{23}$ et leur comparaison avec ceux obtenus par le modèle de Gibson. 
Tableau 3. Caractéristiques du nida obtenues par différents modèles.

\begin{tabular}{cccc}
\hline Nida6 & Samcef & Gibson & Grédiac \\
\hline$E_{1}(\mathrm{MPa})$ & 0,886 & 0,828 & \\
$E_{2}(\mathrm{MPa})$ & 0,886 & 0,828 & \\
$E_{3}(\mathrm{MPa})$ & 1954,97 & 1840 & \\
$\nu_{12}$ & 0,999 & 1 & \\
$\nu_{13}$ & 0 & 0 & \\
$\nu_{21}$ & 0,999 & 1 & \\
$\nu_{23}$ & 0 & 0 & \\
$\nu_{31}$ & 0,33 & 0,33 & \\
$\nu_{31}$ & 0,33 & 0,33 & \\
$G_{12}(\mathrm{MPa})$ & 0,475 & 0,497 & \\
$G_{21}(\mathrm{MPa})$ & 0,17 & & \\
$G_{23}(\mathrm{MPa})$ & 238 & 250 & 250 \\
$G_{32}(\mathrm{MPa})$ & 57 & & \\
$G_{13}(\mathrm{MPa})$ & 389,2 & & 383 \\
$G_{13-\text { mini }}(\mathrm{MPa})$ & & 375 & 375 \\
$G_{13-\text { maxi }}(\mathrm{MPa})$ & & 416,7 & 416,7 \\
$G_{31}(\mathrm{MPa})$ & 0,92 & & \\
\hline
\end{tabular}

Les résultats sont assez proches car l'écart entre Samcef et Gibson est d'environ $1 \%$. Ce module est très important. Il a le même rôle que le module $G_{13}$ dans le comportement des structures sandwiches.

La synthèse de l'ensemble des simulations effectuées (avec les différents modèles) pour la détermination de l'ensemble des caractéristiques du nida est réalisée dans le tableau 3.

\subsubsection{Bilan des simulations et interprétations}

Le nida est considéré comme un matériau orthotrope. Du fait de sa géométrie bien spécifique, il est assez difficile de caractériser son comportement mécanique. Le calcul numérique permet d'avoir une première approche des réactions de matériaux un peu capricieux tels que le nida, qui ne se prêtent pas toujours à des modélisations analytiques simplifiées.

Cette étude a permis de montrer que malgré les difficultés que pose le nida, en utilisant les modélisations analytiques et numériques en parallèle, on arrive à déterminer son comportement. Il est à remarquer que plusieurs modèles numériques sont nécessaires pour déterminer complétement l'ensemble des constantes élastiques.

Le modèle comprenant 40 cellules paraît satisfaire la résolution des modules d'élasticité et des coefficients de Poisson. Il fonctionne bien pour des charges axiales du type traction-compression. Il ne permet pas par contre une évaluation du module de cisaillement $G_{13}$. C'est là que le modèle de Grédiac devient intéressant. Il est surtout fait pour simuler du cisaillement.

On constate que la détermination des modules d'Young $E_{1}$ et $E_{2}$ par les deux méthodes analytique et numérique est correcte car l'écart entre le modèle de Gibson et la simulation numérique est de 0,4\%. Par contre l'erreur sur les coefficients de Poisson est toujours très faible.
Nous avons rencontré cependant un problème dans la détermination de $E_{3}$ qui s'avère assez élevé par rapport au modèle de Gibson (10 \%). Nous sommes cependant moins éloignés que les données des fournisseurs tel que Hexcel [8], qui pour un clinquant d'épaisseur $t=0,018 \mathrm{~mm}$ donnent un module $E_{3}$ de $207 \mathrm{MPa}$, ce qui correspond à un écart de $39 \%$.

Pour ce qui concerne les modules de cisaillement les écarts entre les simulations numériques et les simulations analytiques sont relativement faibles pour les quatre épaisseurs de clinquant que nous avons considérées. Les résultats que nous obtenons, pour un feuillard d'épaisseur $t=0,018 \mathrm{~mm}$, sont très proches des données de Hexcel. En effet l'erreur sur le module de cisaillement $G_{12}$ varie de 3,4 à 4,8\%. Cette erreur assez importante concerne un module de faible valeur donc peu prépondérant dans les propriétés mécaniques des sandwiches. L'erreur sur $G_{13}$ est de $5 \%$, celle sur le module $G_{23}$ est de $12 \%$. Les caractéristiques $G_{21}, G_{31}$ et $G_{32}$ sont obtenues uniquement par simulations numériques et ne peuvent être comparées avec des modèles analytiques inexistants.

Le raffinement des conditions aux limites par couplage des nœuds que nous avons développé, permet de fortement recaler les simulations numériques par rapport au modèle analytique de Gibson.

\section{Simulation de la flexion 3 et 4 points d'une structure sandwich}

\subsection{Simulations analytiques}

Aujourd'hui, il existe deux types d'approches pour ces problèmes. L'approche bidimensionnelle (pour les plaques minces) qui décrit correctement le champ de contraintes lorsqu'on est suffisamment loin des bords. La deuxième approche (tridimensionnelle) permet de décrire les effets d'extrémités.

Les premières théories développées furent celles du type Kirchhoff-Love [9], ce sont des théories de premier ordre, ainsi qualifiées en raison de leur dépendance linéaire suivant la surface moyenne. Ce sont des théories de flexion, applicables essentiellement à des structures minces, pour lesquelles les effets de flexion (correspondant aux rotations des fibres autour des tangentes à la surface de référence) prédominent par rapport à ceux dus au cisaillement.

Un autre type d'approche est envisagé par ReissnerMindlin $[10,11]$. Cette approche également linéaire en $z$ prend en compte les effets du cisaillement transverse et notamment sa continuité à la traversée des interfaces. Ces résultats sont applicables à des structures épaisses ou non.

Touratier propose un nouveau type d'approche [12]. L'objectif est de mettre en place une théorie simple mais raffinée, permettant la prise en compte du cisaillement transverse, pour des structures composites multicouches, épaisses ou non. La nouveauté, par rapport aux approches antérieures, réside en l'introduction d'une fonction sinusoïdale pour représenter la part due au cisaillement dans le champ de déplacement. 
Le travail présenté dans cette section a pour but d'établir une base de données comparative entre les résultats fournis par les simulations numériques sur le logiciel de calcul par éléments finis Samcef [6], les modèles analytiques à l'aide du logiciel de calcul formel Mathematica [13] et les résultats expérimentaux par jauges et caméras CCD $[14,15]$; on peut citer également les travaux basés sur la méthode des grilles [16].

\subsubsection{Cinématique}

Avant d'aborder les principaux développements analytiques, il nous paraît nécessaire de citer quelques travaux importants dans l'évolution des théories de plaques, sans toutefois être exhaustif, étant donné le nombre considérable de travaux sur la question, voir par exemple Touratier [12].

On se limite ici aux modèles ayant cinq déplacements généralisés (trois translations et deux rotations) et à déformation nulle suivant l'épaisseur. On peut résumer tous ces modèles par le champ de déplacement suivant :

$$
U=\left\{\begin{array}{l}
u_{\alpha}=u_{\alpha}-z \cdot w_{, \alpha}+f(z) \cdot \gamma_{\alpha} \\
u_{3}=w
\end{array}\right.
$$

avec

$$
\gamma_{\alpha}=\omega_{\alpha}+w_{, \alpha}, \quad \alpha \in\{1,2\}
$$

où $u_{1}, u_{2}, w, \gamma_{1}$ et $\gamma_{2}$ sont des fonctions de $x, y \cdot u_{1}$ et $u_{2}$ représentent les déplacements de membrane, $w$ la flèche, $\gamma_{1}$ et $\gamma_{2}$ les déformations de cisaillement transverse dans le plan moyen, $w_{1}$ et $w_{2}$ les rotations de la normale autour du plan moyen par rapport à 1 et 2 respectivement. $f(z)$ est une fonction définie de telle façon que sa dérivée représente la répartition des contraintes de cisaillement transverse dans l'épaisseur de la plaque. Le choix de la fonction $f(z)$ influe directement sur le raffinement de la théorie, ce qui permet de distinguer les différentes théories :

- $f(z)=0$ Kirchhoff-Love

- $f(z)=z$ Reissner- Mindlin

- $f(z)=z \cdot\left(1-\frac{4 z^{2}}{3 h^{2}}\right)$ Reddy

- $f(z)=\frac{h}{\pi} \cdot \sin \left(\frac{\pi z}{h}\right)$ Touratier

\subsection{2 Équations d'équilibre et conditions aux limites}

Les plaques étudiées sont des sandwiches à symétrie miroir; on aura alors découplage des effets de membrane des effets de flexion. Dans le cas statique les équations

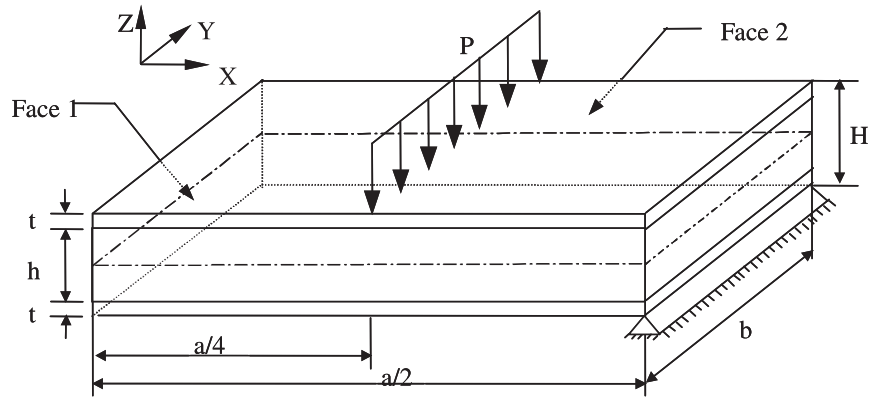

Fig. 5. Plaque sandwich en flexion 4 points.

d'équilibre s'écrivent $\forall$ les champs de déplacements virtuels $\gamma_{1}^{*}, \gamma_{2}^{*}$ et $w^{*}[17]$.

$$
\begin{aligned}
0= & -\left(E_{12}+2 \cdot E_{66}\right) \cdot w_{, 112}-E_{22} \cdot w_{, 222}+\left(\tilde{D}_{12}\right. \\
& \left.+\tilde{D}_{66}\right) \cdot \gamma_{1,12}+\tilde{D}_{66} \cdot \gamma_{2,11}+\tilde{D}_{22} \cdot \gamma_{2,22}-\tilde{A}_{44} \cdot \gamma_{2} \\
0= & -E_{11} \cdot w_{, 111}-\left(E_{12}+2 \cdot E_{66}\right) \cdot w_{, 122}+\tilde{D}_{11} \cdot \gamma_{1,11} \\
& +\left(\tilde{D}_{12}+\tilde{D}_{66}\right) \cdot \gamma_{2,12}+\tilde{D}_{66} \cdot \gamma_{1,12}-\tilde{A}_{55} \cdot \gamma_{1} \\
0= & -D_{11} \cdot w_{, 111}-2 \cdot\left(D_{12}+2 \cdot D_{66}\right) \cdot w_{, 1122} \\
& -D_{22} \cdot w_{2222}+E_{11} \cdot \gamma_{1,111}+\left(E_{12}+2 \cdot E_{66}\right) \cdot\left(\gamma_{1,122}\right. \\
& \left.+\gamma_{2,112}\right)+E_{22} \cdot \gamma_{2,222}+q
\end{aligned}
$$

Pour une plaque rectangulaire dont le système d'axes est représenté figure 5, les conditions aux limites naturelles $\forall$ les champs de déplacements virtuels $\gamma_{1}^{*}, \gamma_{2}^{*}$, $w^{*}$ et $w_{, n}^{*}$ sur les bords $y=-b / 2$ et $y=b / 2$ sont :

$$
\begin{aligned}
0= & 2 \cdot E_{66} \cdot w_{, 12}-\tilde{D}_{66} \cdot \gamma_{1,2}-\tilde{D}_{66} \cdot \gamma_{2,1}+\tilde{C}_{1} \\
0= & E_{12} \cdot w_{, 11}+E_{22} \cdot w_{, 22}-\tilde{D}_{12} \cdot \gamma_{1,1}-\tilde{D}_{22} \cdot \gamma_{2,2}+\tilde{C}_{2} \\
0= & \left(D_{12}+4 \cdot D_{66}\right) \cdot w_{, 112}+D_{22} \cdot w_{, 222}-\left(E_{12}\right. \\
& \left.+2 \cdot E_{66}\right) \cdot \gamma_{1,12}-2 \cdot E_{66} \cdot \gamma_{2,11}-E_{22} \cdot \gamma_{2,22} \\
& +T_{z}+C_{1,1} \\
0= & -D_{12} \cdot w_{, 11}-D_{22} \cdot w_{, 22}+E_{22} \cdot \gamma_{2,2}+E_{12} \cdot \gamma_{1,1}-C_{2}
\end{aligned}
$$

où

$$
\begin{gathered}
\left(A_{i j}, \tilde{A}_{i j}, B_{i j}, \tilde{B}_{i j}, D_{i j}, \tilde{D}_{i j}, E_{i j}\right)= \\
\int_{-h / 2}^{h / 2}\left[1, f^{\prime}(z)^{2}, z, f(z), z^{2}, f(z)^{2}, z \cdot f(z)\right] Q_{i j} \mathrm{~d} z \\
\left(T_{\alpha}, C_{\alpha}, \tilde{C}_{\alpha}\right)=\int_{-h / 2}^{h / 2}(1, z, f(z)) \cdot F_{\alpha} \cdot \mathrm{d} z \\
T_{z}=\int_{-h / 2}^{h / 2} F_{3} \cdot \mathrm{d} z
\end{gathered}
$$

\subsubsection{Problème aux limites}

L'introduction des solutions de Lévy dans les trois équations d'équilibre (9) et la transformation de 

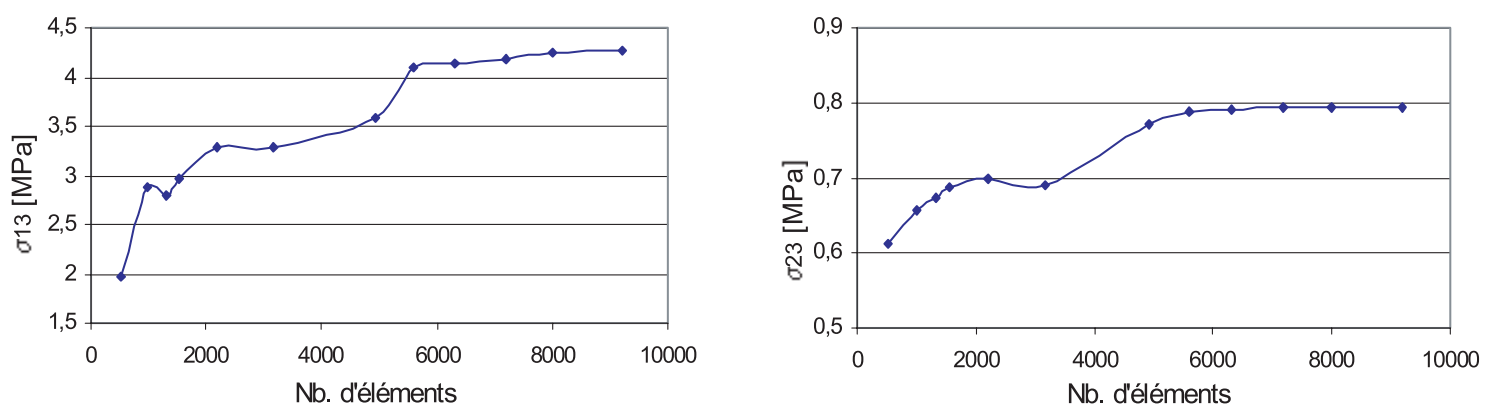

Fig. 6. Convergence des contraintes $\sigma_{13}$ et $\sigma_{23}$.

Tableau 4. Comparaison des déplacements entre les modèles analytiques et Samcef.

\begin{tabular}{lcccccc}
\hline \multirow{2}{*}{ Modèle } & $w[\mathrm{~mm}]$ & $\begin{array}{c}\text { Erreur } \\
{[a / 2,0,0]}\end{array}$ & $\begin{array}{c}u_{1}[\mathrm{~mm}] \\
{\left[3^{*} a / 4,0, H / 2\right]}\end{array}$ & $\begin{array}{c}\text { Erreur } \\
\%\end{array}$ & $\begin{array}{c}u_{2}[\mathrm{~mm}] \\
{\left[3^{*} a / 4,4^{*} b / 10, H / 2\right]}\end{array}$ & $\begin{array}{c}\text { Erreur } \\
\%\end{array}$ \\
\hline Samcef & $-3,41$ & 4,4 & $-0,102$ & 0,9 & 0,0069 & 1,7 \\
Mindlin & $-2,94$ & 10,1 & $-0,091$ & 11,3 & 0,0065 & 7,6 \\
Reddy & $-3,24$ & 0,8 & $-0,103$ & 0,07 & 0,0071 & 0 \\
Touratier & $-3,27$ & Ref. & $-0,103$ & Ref. & 0,0071 & Ref. \\
\hline
\end{tabular}

ces équations aux dérivées partielles en un système d'équations différentielles du premier ordre, amène la réorganisation du système sous la forme suivante [17] :

$$
A \cdot X^{\prime}+B \cdot X+C=0
$$

Les solutions de Lévy permettent de prendre en compte des conditions d'appuis simples à deux extrémités de la plaque. Les deux bords restants peuvent être encastrés, libres ou simplement appuyés.

Les conditions aux limites de type cinématique assurent les conditions d'encastrement, les conditions aux limites naturelles assurent les conditions de bords libres et chargés, enfin une combinaison de ces deux types de conditions aux limites assure les conditions d'appuis simples.

La méthode de résolution que nous avons développée a été implantée dans le logiciel de calcul formel Mathematica.

\subsection{Simulations numériques}

L'absence de solution exacte pour notre problème nous a amené à réaliser des simulations numériques. Nous avons exploité un modèle numérique sur le code de calcul Samcef [6].

L'étude de la flexion 3 et 4 points a été effectuée en 3D (Fig. 5) et le choix de l'élément s'est porté sur un élément quadrilatère solide à huit nouds et six degrés de liberté par noud (hypothèse de Mindlin).

En raison des symétries de la forme et du chargement, le modèle de base est constitué d'un quart de la plaque. Pour rendre compte des symétries, plusieurs tests ont été nécessaires, les conditions aux limites appliquées pour les deux cas de flexion sont : au niveau de l'appui, annulation de la translation $U_{3}$ et au niveau de la symétrie sur la face 1 , annulation de la translation $U_{1}$ et des rotations $\theta_{2}$ et $\theta_{3}$ et sur la face 2 , annulation de la translation $U_{2}$ et des rotations $\theta_{1}$ et $\theta_{3}$ (Fig. 5).

La plaque sandwich est constituée des peaux en aluminium et du cœur en nids d'abeilles en aluminium dont les dimensions sont :

La longueur $a=510 \mathrm{~mm}$, la largeur $b=100 \mathrm{~mm}$, l'épaisseur $H=15,8 \mathrm{~mm}$ avec celle de la peau $t=0,8 \mathrm{~mm}$ et du cœur $h=14,2 \mathrm{~mm}$, la charge appliquée est linéique sur toute la largeur.

Les caractéristiques mécaniques de la peau sont [8] :

$$
E=73087 \mathrm{MPa}, G=23305 \mathrm{MPa}, \nu=0,33 .
$$

Les caractéristiques mécaniques du nid d'abeilles sont [18] :

$E_{1}=4 \mathrm{MPa}, \quad G_{12}=2 \mathrm{MPa}, \quad \nu_{12}=0,25$

$E_{2}=6 \mathrm{MPa}, \quad G_{23}=47 \mathrm{MPa}, \quad \nu_{23}=0,02$

$E_{3}=220 \mathrm{MPa}, G_{13}=104 \mathrm{MPa}, \quad \nu_{13}=0,02$

Les charges appliquées sur la plaque sandwich sont linéiques et uniformes avec des valeurs de $1000 \mathrm{~N}$ en flexion 3 points et $700 \mathrm{~N}$ en flexion 4 points.

\subsubsection{Convergence du maillage}

La modélisation numérique par éléments finis nécessite l'étude de convergence du maillage afin de valider les résultats obtenus par approximation.

Compte tenu des symétries du problème, nous avons effectué la modélisation d'un quart de plaque. La convergence est acquise à 5600 éléments répartis en cinquante éléments suivant 1, dix éléments dans l'épaisseur du cour, trois éléments dans l'épaisseur de la peau et sept éléments suivant 2 .

\subsubsection{Résultats}

Les résultats obtenus avec les différents modèles analytiques et numériques sont regroupés dans le tableau 4 
Tableau 5. Comparaison des contraintes entre les modèles analytiques et Samcef.

\begin{tabular}{lcccccc}
\hline \multirow{2}{*}{ Modèle } & $\begin{array}{c}\sigma_{11}[\mathrm{MPa}] \\
{\left[3^{*} a / 4,0, H / 2\right]}\end{array}$ & $\begin{array}{c}\text { Erreur } \\
\%\end{array}$ & $\begin{array}{c}\sigma_{23}[\mathrm{MPa}] \\
{\left[a / 2,4^{*} b / 10, h / 2\right]}\end{array}$ & $\begin{array}{c}\text { Erreur } \\
\%\end{array}$ & $\begin{array}{c}\sigma_{13}[\mathrm{MPa}] \\
{\left[3^{*} a / 4,4^{*} b / 10,(h+t) / 2\right]}\end{array}$ & $\begin{array}{c}\text { Erreur } \\
\%\end{array}$ \\
\hline Samcef & $-39,72$ & 1,1 & 0,463 & 7,3 & 2,25 & 8,6 \\
Mindlin & $-39,12$ & 0,4 & 0,391 & 9,5 & 2,43 & 1,1 \\
Reddy & $-39,27$ & 0,02 & 0,468 & 8 & 2,73 & 10,9 \\
Touratier & $-39,28$ & Ref. & 0,432 & Ref. & 2,46 & Ref. \\
\hline
\end{tabular}

Tableau 6. Résultats pour la flèche $w(a / 2)$ obtenus pour la plaque en flexion 3 points en fonction de la charge.

\begin{tabular}{ccccccc} 
& $w(a / 2)[\mathrm{mm}]$ & Erreur & $w(a / 2)[\mathrm{mm}]$ & $\begin{array}{c}\text { Erreur } \\
\%(a / 2)[\mathrm{mm}]\end{array}$ & $\begin{array}{c}\text { Erreur } \\
\%\end{array}$ \\
\hline CCD & à $250 \mathrm{~N}$ & $\%$ & à $300 \mathrm{~N}$ & $\%$ & à 350 N & $\%$ \\
Samcef & $-1,15$ & 4,7 & $-1,34$ & 1,7 & $-1,579$ & 2,7 \\
Mindlin & $-1,127$ & 2,6 & $-1,352$ & 2,6 & $-1,578$ & 2,7 \\
Reddy & $-1,067$ & 2,8 & $-1,281$ & 2,8 & $-1,494$ & 2,8 \\
Touratier & $-1,096$ & 0,2 & $-1,315$ & 0,2 & $-1,534$ & 0,2 \\
\hline
\end{tabular}

pour les déplacements et dans le tableau 5 pour les contraintes.

Dans notre étude nous nous sommes intéressés à un sandwich à cœur souple et à peaux très rigides. Qualitativement, on a été amené à comparer les résultats des différents modèles analytiques avec ceux obtenus par la simulation numérique sur Samcef.

L'erreur sur la flèche $w$ dans le cas flexion 3 points, pour le modèle Mindlin est de l'ordre de $10 \%$ par rapport à la solution de référence modèle Sinus (Touratier), par contre l'erreur pour le modèle de Reddy et Samcef est moins importante, et pour le cas de la flexion 4 points l'erreur est plus faible pour tous les modèles.

Pour les déplacements $U_{1}$ et $U_{2}$ le modèle de Mindlin donne une erreur (dans le cas de la flexion 3 points) plus importante $(11 \%)$ que les autres modèles.

L'amplitude des contraintes de cisaillement transverse ne diffère pas notablement d'un modèle à l'autre, à part le modèle de Mindlin où la contrainte de cisaillement est constante dans l'épaisseur. On note cependant que pour le modèle de Reddy le cisaillement transverse $\sigma_{13}$ est plus important dans les peaux que pour le modèle Sinus.

On remarque que les résultats de Samcef sont cohérents avec les autres modèles et l'erreur ne dépasse pas $9 \%$ par rapport à la référence (Touratier).

\subsection{Validation expérimentale}

Un grand nombre de modèles de plaques et de poutres a été développé pour décrire la répartition des contraintes dans les structures sandwiches et notamment la composante de cisaillement comme il a été décrit à la section 3.1.1. Cependant, les travaux expérimentaux concernant l'étude des champs cinématiques dans l'épaisseur des structures sandwiches restent peu nombreux pour valider les différentes théories [19,20]. Le but de ce travail est de mesurer les champs de déplacement et de déformation sur la surface latérale d'une poutre sandwich soumise à des sollicitations de flexion trois et quatre points. On utilise pour cela deux méthodes optiques l'une basée sur

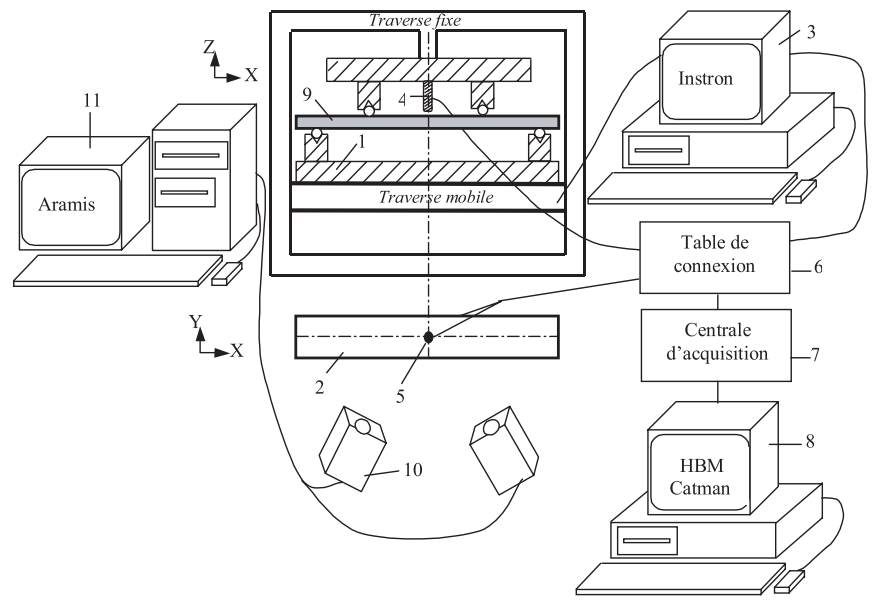

Fig. 7. Banc d'essai expérimental.

la corrélation d'images, l'autre sur l'interférométrie speckle. Il est alors possible de comparer les profils des déplacements obtenus pour différentes structures sandwiches avec ceux développés dans la section précédente.

\subsubsection{Description des essais}

Le banc expérimental (Fig. 7) est constitué d'un support $\{1\}$ sur lequel est bridée la plaque $\{2\}$. Le contrôle de la valeur de la charge s'effectue à l'aide du logiciel Instron $\{3\}$. Un capteur de déplacement Tr102 \{4\} est fixé sur le support supérieur. Deux rosettes à $45^{\circ}\{5\}$ sont collées sur les faces inférieure et supérieure de l'éprouvette et sont branchées sur une table de connexion $\{6\}$. L'acquisition est réalisée à l'aide d'une centrale HBM UPM60 \{7\} pilotée par un ordinateur PC disposant du logiciel Catman $\{8\}$. Une couche grise de peinture $\{9\}$ est appliquée sur le côté de la plaque. Deux caméras CCD $\{10\}$ permettent d'acquérir des images numérisées. Ces images sont ensuite analysées à l'aide du logiciel Aramis $\{11\}$ [7].

Deux systèmes de mesure ont été utilisés lors de cette opération : le système Aramis basé sur la corrélation 


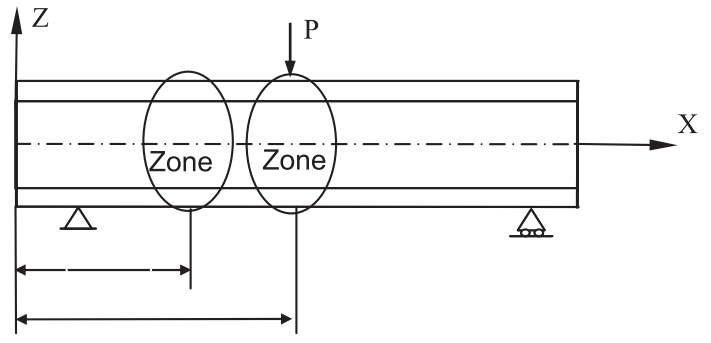

Fig. 8. Flexion 3 points du sandwich.

d'images en 2D nous a permis de mesurer le déplacement transversal «w» de la poutre sandwich. Il n'a pas permis le calcul des déformations de l'âme car les discontinuités formées par les arêtes du nid d'abeilles empêchent la propagation du calcul lors du traitement des images. Pour compléter les mesures nous avons utilisé le deuxième système basé sur l'interférométrie speckle.

Les techniques d'analyse ponctuelles par corrélation d'images sont extrêmement sensibles et permettent une caractérisation mécanique très efficace. Cependant le seuil de leur utilisation doit être supérieur à $0,1 \%$ en déformation et les surfaces de mesure doivent être régulières. Le besoin de compléter cette gamme de mesure s'est rapidement fait sentir. C'est pourquoi nous avons utilisé de nouvelles techniques d'imagerie qui permettent en quelques minutes de cartographier le champ de déplacement et par conséquence de calculer des déformations (pour des seuils inférieurs à $0,1 \%$ ) et des contraintes sur des structures qui peuvent présenter certaines irrégularités de forme (comme c'est le cas de l'âme du sandwich étudié). Ces techniques sont basées sur l'interférométrie de speckle. Elles consistent à imager la surface de l'échantillon en lumière cohérente sur un capteur CCD. Dans ces conditions une structure granulaire (le speckle) souvent assimilée à du bruit se superpose à l'image. Cette structure est la signature ou « empreinte digitale » de la surface de l'échantillon $[21,22])$. Lorsque la surface se déforme la structure du speckle change et une analyse de son évolution permet de mesurer la déformation du composant. L'analyse se fait après l'acquisition de l'image et traitement informatique [23]. Il est alors possible de mesurer les déplacements et ensuite par calcul d'accéder aux déformations et aux contraintes dans les structures.

\subsubsection{Mesure du champ de déplacement}

La corrélation d'image a été utilisée pour mesurer le déplacement transverse le long de la ligne centrale suivant $x$ de la plaque sandwich (Fig. 8). Les résultats sont ensuite comparés avec ceux issus des méthodes analytiques et des simulations par éléments finis (Tab. 6). La caméra numérique utilisée à une résolution de $1280 \times 1024$, le logiciel ARAMIS 2D (version 4.7.4) permet le traitement d'images et par conséquent la mesure des déplacements de l'ordre du micron. Le calcul des déformations est assuré avec une résolution d'environ 500-1000 $\mu \mathrm{m} \cdot \mathrm{m}^{-1}(0,05-$ $0,1 \%)$.

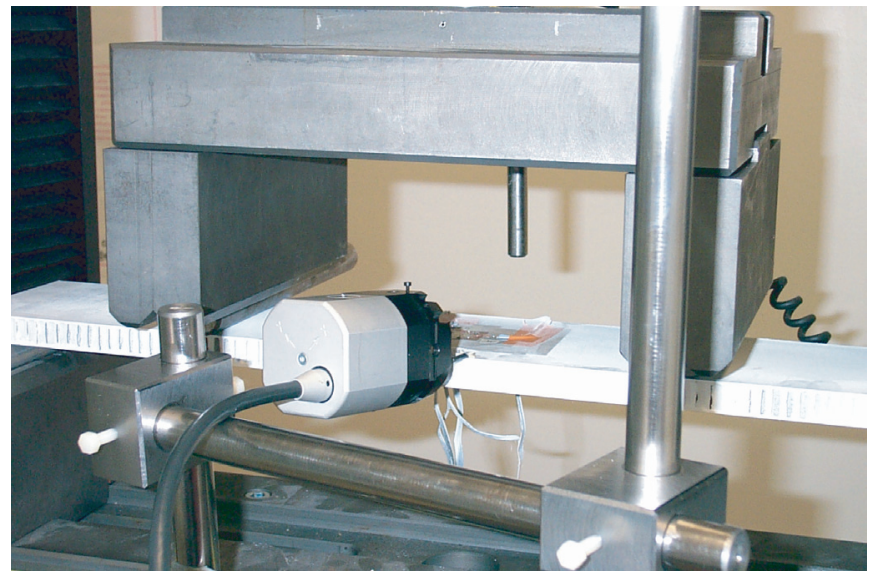

Fig. 9. Banc d'essai de la flexion 4 points, équipé du système ESPI (Microstar).

Tableau 7. Déformations obtenues en flexion 3 points pour $350 \mathrm{~N}$ sur la zone 1.

\begin{tabular}{ccccc}
\hline Charge & $\varepsilon_{11}(Z=H / 2)$ & $\begin{array}{c}\text { Erreur } \\
\%\end{array}$ & $\varepsilon_{22}(Z=H / 2)$ & $\begin{array}{c}\text { Erreur } \\
\%\end{array}$ \\
350 N & & 1,3 & 170 & 2,4 \\
\hline Microstar & -555 & $1,3,4$ & 172,9 & 4,1 \\
Samcef & -543 & 3,4 & 10,9 \\
Mindlin & $-538,4$ & 4,2 & 184,2 & 1,6 \\
Reddy & $-561,9$ & 0,07 & 163,4 & REF. \\
Touratier & $-562,3$ & REF. & 166 & \\
\hline
\end{tabular}

Tableau 8. Déformations obtenues en flexion 3 points pour $350 \mathrm{~N}$ sur la zone 2.

\begin{tabular}{ccccc}
\hline $\begin{array}{c}\text { Charge } \\
350 \mathrm{~N}\end{array}$ & $\varepsilon_{11}(Z=H / 2)$ & $\begin{array}{c}\text { Erreur } \\
\%\end{array}$ & $\varepsilon_{22}(Z=H / 2)$ & $\begin{array}{c}\text { Erreur } \\
\%\end{array}$ \\
\hline Microstar & -270 & 1,3 & 83 & 11,5 \\
Samcef & $-272,6$ & 0,4 & 83,2 & 11,8 \\
Mindlin & $-273,2$ & 0,2 & 83,5 & 12,1 \\
Reddy & $-273,6$ & 0,01 & 73,8 & 1 \\
Touratier & $-273,6$ & Ref. & 74,5 & Ref. \\
\hline
\end{tabular}

\subsubsection{Mesure des déformations $\varepsilon_{1}$ et $\varepsilon_{2}$ avec le microstar}

L'interférométrie speckle a été utilisée pour mesurer le champ de déformation dans deux zones. Une zone se situant sous l'appui central et une autre entre les deux appuis. Les résultats sont ensuite comparés avec ceux issus des méthodes analytiques et des simulations par éléments finis (Tab. 7). Le système ESPI appelé (Microstar) à 4 sources laser à $90^{\circ}$ et une caméra dont la résolution de $768 \times 582$ pixels permet la mesure des déplacements au dizième de micron et le calcul des déformations avec une résolution de $10 \mu \mathrm{m} . \mathrm{m}^{-1}$. La distance de travail est de $32 \mathrm{~mm} \pm 4 \mathrm{~mm}$ et la surface mesurée est de $34 \times 26 \mathrm{~mm}$. La résolution spatiale est de $0,4-0,5 \mathrm{~mm}(1$ pixel $=45 \mu \mathrm{m})$.

L'objectif des développements de la section 3.3 est de valider les résultats obtenus par les différents modèles (analytiques et numériques) par une méthode expérimentale.

Nous avons utilisé des méthodes de mesure sans contact (corrélation d'image et interférométrie speckle) pour les déplacements et les déformations. Ces méthodes 
ont été dans une première phase étalonnées d'abord sur des matériaux isotropes afin de juger de leur précision en les comparant avec des capteurs traditionnels (jauges d'extensomètrie) capteur de déplacement (LVDT). Dans une seconde phase nous avons étendu leur utilisation aux matériaux sandwiches.

Nous avons montré les limites du système de mesure par corrélation d'image dans la mesure des déformations à cause des singularités liées à la forme de l'âme du sandwich, ce qui a rendu nécessaire l'utilisation de l'interferométrie speckle. La corrélation entre les différents modèles est correcte.

\section{Conclusion}

La connaissance des propriétés mécaniques est la première étape dans la compréhension des matériaux sandwiches. Nous avons développé une méthode d'homogénéisation numérique qui a permis la détermination de la matrice de rigidité tridimensionnelle du nid d'abeilles à structure hexagonale utilisé dans la confection des matériaux sandwiches étudiés. La corrélation entre les modèles de la littérature et les résultats obtenus par simulation sont en bon accord.

L'étude comparative des principaux modèles théoriques de plaques en statique a permis d'analyser les performances des modèles à base Sinus. Une hiérarchie a été dégagée après une analyse sur des configurations de composites sévères; «sandwiches épais ». Une analyse des effets de bord a été également réalisée.

La rareté des solutions exactes pour les problèmes de composites « sandwiches » chargés transversalement et sous liaisons sévères nous a conduit à évaluer des modèles analytiques basés sur des approximations du champ de déplacement. Successivement ont été testés les modèles à couche équivalente (Kirchhoff-Love, Reissner-Mindlin, Reddy et Touratier) et des modèles éléments finis (Samcef) dans le souci de mieux représenter les différents champs aux interfaces (point essentiel pour les conditions de transfert de charge dans les structures sandwiches).

Nous avons mis en ouvre deux techniques de mesure, l'une basée sur la corrélation d'images, l'autre sur l'interférométrie speckle, qui ont donné de bons résultats en comparaison avec les modèles analytiques et numériques. Ces techniques ouvrent des horizons prometteurs en matière d'analyse des contraintes.

\section{Références}

[1] L.J. Gibson \& M.F Ashby, Cellular solids, structure and properties, Pergamon Press, 1988

[2] L.J. Gibson, Hierarchical cellular materials, Materials research society, Fall Meeting, 1991

[3] M. Grédiac, A finite element study of the transverse shear in honeycomb cores, Int. J. Solids structures 30 (1993) $1777-1788$
[4] C.C. Chamis, R.A. Aiello, L.N. Murthy, Fiber composite sandwich thermostructural behaviour: Computational simulation, J. composites Tech. Research 10 (1988) 93-99

[5] S. Kelsey, R.A. Gellatly, B.W. Clark, The shear modulus for foil honeycomb core, Aircraft engineering, 1958, pp. 294-302

[6] Samcef, version 7.1, User's manuals, 1997

[7] L. Albachi, M. Karama, Caractérisation des propriétés des nids d'abeilles par une méthode numérique, Premier colloque interdisciplinaire sur les matériaux en France, Tours, 21-25 octobre, 2002

[8] Hexcel, Design of sandwich structures, Structural Materials Handbook, Section IV, 1994

[9] A.E.H. Love, A Treatise on the mathematical theory for elasticity 4th Ed., Dover, New York, 1927

[10] E. Reissner, The effect of transverse shear deformation on the bending of elastic plates, J. Appl. Mech. 12 (1945) $69-77$

[11] R.D. Mindlin, Influence of rotatory inertia and shear on flexural motions of isotropic elastic plates, J. Appl. Mech. 18 (1997) 31-38

[12] M. Touratier, Un modèle simple et efficace en mécanique des structures composites, C. R. Acad. Sci. Paris 309 (1989) 933-938

[13] Mathematica, version 2, A system for doing mathematics by computer, Stephen Wolfram, inc., 1991

[14] D. Bergmann, R. Ritter, 3D Deformation Measurement in small Areas based on Grating Method and Photogrammetry, 1994

[15] M. Karama, L. Albachi, S. Mistou, Photogrammetry method for measuring the 3D deformation, International Workshop on Video-Controlled Materials Testing- Ecole de Mines de Nancy 1999, pp. 141-144

[16] L. Dufort, M. Grediac, Y. Surrel, Experimental evidence of the cross section warping in short composite beams under three point bending, Composite Structures, Elsevier 2001, 51, pp. $37-47$

[17] A. Idlbi, Comparaisons de théories de plaques et estimation de la qualité des solutions dans la zone de bord, Thèse de l'ENSAM Paris, 1995

[18] F. Bonac, Matériaux composites, comportement mécanique et analyse des structures, éditions Masson, 1996

[19] Y. Surrel, Moiré and grid methodes: a signal-processing approach, Ryszard, J. Pryputmiewicz et J. Stupnicki, Interferometry, photomechanics, Proc. Soc. Pho-Opt. Instrum. Eng. 2342 (1994) 213-220

[20] Y. Surrel, Design of algorithms for phase measurements by the use of phase-stepping, Appl. Opt. 35 (1996) 51-60

[21] P. Synnergren, M. Sjödahl, A stereoscopic digital speckle photography system for 3D displacement field mesurements, Optics and Lasers in Engin. 31 (1999) 425-443

[22] A. Ettemeyer, Combination of 3D deformation and shape mesurement by electronic speckle-pattern interferometry for quantitive strain-stress analysis, Opt. Eng. 39 (2000) $212-215$

[23] J. Tyson, R. Wegner, A. Ettemeyer, Noncontact total strain mesurement for failure prediction, Materials Evaluation (1999) 576-580 